\title{
Médecine, philosophie et révolution scientifique. Une étude bibliographique
}

Claire Crignon, Delphine Antoine-Mahut

\section{Summary}

This article examines the place that has been reserved for medicine in the historiography of the sciences. More precisely, it focuses on the motifs that have lead historians of science to grant only a minor role to medicine within the movement commonly designated by the notion of the "scientific revolution". Among those motifs, the persistent and late application of teleological schemas in the thinking of the biological and the difficulties in "mathematizing" anatomy are often invoked. Starting with an overview of the critical literature on the topic, this bibliographical essay shows how the situation has changed over the last decades. The opposition between, on the one hand, the physical sciences founded on a model of mechanistic explanation of nature and, on the other hand, the life sciences that remained guided by a finalist mode of thinking are today much put into question. What we find today is more open reflection on the diversity of "models" for understanding the living, and on how to integrate them into more complex schemas than those that simply oppose mechanism and teleology. The essay is finally based on discussions and debates among medical doctors and philosophers in the modern period, and insists on the importance of studying this "medico-philosophical" tradition in order to avoid reconstructing a posteriori a mythical history that trends to consecrate a single model of rationality.

Keywords: Medicine, philosophy and historiography of life sciences, scientific revolution

Claire Crignon, Université Paris-Sorbonne IV, UFR de Philosophie et Sociologie, 1 rue Victor Cousin, F-75005, Paris (claire.crignon@paris-sorbonne.fr). Delphine Antoine-Mahut, Ecole Normale Supérieure de Lyon, 15 parvis René Descartes, Bureau R115, F-69007 Lyon (delphineantoine-mahut@ens-lyon.fr) 


\section{Résumé}

Cet article examine la place qui a été réservée à la médecine dans l'historiographie des sciences. Il s'intéresse plus particulièrement aux motifs qui ont pu conduire à assigner à la médecine un rôle mineur au sein de ce mouvement de transformation des sciences communément désigné par le concept de «révolution scientifique». Parmi ces motifs, la persistance tardive, dans la pensée biologique, de schèmes d'explication téléologique et la difficulté à «mathématiser» l'anatomie sont souvent invoqués. A partir d'un passage en revue de la littérature critique sur ce sujet, cet essai bibliographique montre comment la situation a changé depuis quelques décennies. L'opposition entre les sciences physiques fondées sur un modèle d'explication mécaniste de la nature, d'une part, et les sciences du vivant guidées par un mode de pensée finaliste, d'autre part, se trouve désormais largement remise en question. C'est une réflexion plus ouverte qui se fait jour sur la diversité des «modèles» pour appréhender le vivant et sur la manière de les intégrer au sein de schémas plus complexes que la simple opposition entre mécanisme et téléologie. L'essai s'appuie finalement sur les discussions et les débats entre médecins et philosophes à l'époque moderne et insiste sur la nécessité de partir de l'étude de cette tradition «médico-philosophique» si l'on veut éviter de reconstituer a posteriori une histoire mythique consacrant le triomphe d'un modèle unique de rationalité.

\section{Médecine et révolution scientifique}

Quelle place réserver à la médecine dans les récits de ce que l'historiographie des sciences a désigné sous l'étiquette de «révolution scientifique»? Comme le soulignait Harold J. Cook en 1990, cette question a pu être considérée comme l'une des plus épineuses pour l'historien des sciences: «[...] the Relationship between medicine and the intellectual movement conventionally known as the Scientific Revolution have vexed historians for decades.» ${ }^{1}$

Plusieurs éléments d'explication peuvent être mentionnés. Tout d'abord, le statut même de la médecine, art fondé sur l'observation empirique, renvoyant d'abord à une pratique et à un objectif thérapeutique (guérir), avant de se définir comme une science reposant sur une connaissance théorique. ${ }^{2}$

1 Cook 1990, 397.

2 Comme le note Cook 1990, 399, cette double dimension de la médecine se traduisait dans l'anglais de la période moderne par l'usage de deux termes différents: medicine pour l'art de guérir et physic pour la médecine comme savoir et partie de la philosophie naturelle. 
Considérée avant tout comme un savoir «appliqué», la médecine aurait joué un rôle mineur dans la «révolution scientifique», comparativement aux sciences physiques «pures». Ensuite, la «persistance» lancinante de modèles anciens du corps (particulièrement de la théorie d'inspiration scolastique et galénique des éléments et des humeurs), ${ }^{3}$ considérée comme un «obstacle épistémologique» à un véritable progrès en médecine. Ou encore, la proximité de la médecine avec des savoirs considérés comme «occultes», comme l'alchimie, signe de son incapacité à accéder à une forme de rationalité satisfaisante pour A. Koyré, C. Singer ou encore H. Butterfield ${ }^{4}$ ou, plus précisément encore selon Richard S. Westfall, des difficultés qu'elle rencontre à adopter le modèle d'explication mécaniste de la nature qui se diffuse largement à partir du XVII ${ }^{\mathrm{e}}$ siècle. ${ }^{5}$

Ce type d'interprétation a pu conduire de manière assez radicale à exclure la médecine du champ de la révolution scientifique ou à ne lui reconnaître qu'un rôle mineur. On mentionnera ici deux exemples de cette tendance. L'ouvrage classique de E. J. Dijksterhuis, The Mechanization of the World Picture. Pythagoras to Newton ${ }^{6}$, n'accorde quasiment aucune place à la médecine, sinon en mentionnant la figure de William Gilbert et de ses recherches sur le magnétisme (De Magnete, Magneticisque Corporibus, et de Magno Magnete Tellure; Physiologia Nova, Plurimis et Argumentis, et Experimentis Demonstrata, London, 1600) et en soulignant l'absence totale de relation entre la pensée de ce dernier et ce qui pourrait s'apparenter à un effort pour mathématiser la nature ou produire des expériences fondées sur des mesures quantitatives (p. 391). Dix ans plus tard, dans un ouvrage sur l'histoire des idées scientifiques, Charles C. Gillispie considère que la pensée de Vésale, contrairement à celles de Copernic et de Galilée, n'a en rien contribué à changer la conception que l'homme se fait du monde ou de lui-même. Les «sciences de la vie» n'occupent selon lui qu'une «place subordonnée» au sein du vaste mouvement de transformation qui aboutit à la naissance de la «science moderne». Contrairement à ce qui se passe en physique avec Kepler, Galilée et Newton, la médecine se serait montrée incapable de subsumer, sous un ensemble de lois unifiées et à l'intérieur d'une seule science mathématique, l'ensemble des phénomènes recueillis ou observés par le biais de l'histoire naturelle et des pratiques d'observation et de soin. ${ }^{7}$

3 Sur cette «persistance» du modèle galénique interprétée comme le signe d'un «combat d'arrière garde», voir Grmek 1997, 157-158. Sur cette question, voir aussi Temkin 1973.

4 Koyré 1966 et 1968.

5 Westfall 1971, 67-68 et 91.

6 Dijksterhuis 1950.

7 Cf. Gillispie 1960, 58-59. 
Même si elle adopte des formes variées en fonction des corpus et des auteurs, une telle dévaluation de la place des découvertes médicales semble ainsi résulter de l'établissement d'un lien intrinsèque entre l'idée de révolution scientifique et l'adoption d'un modèle mécaniste de la nature, pensé de manière réductionniste comme susceptible de s'appliquer de façon heuristique à l'ensemble des phénomènes.

Dans un tel cadre interprétatif, il n'est possible de parler de «révolution scientifique» qu'à partir du moment où «disparaissent - ou [sont] vigoureusement chassées - de la pensée scientifique toutes les considérations invoquant la valeur, la perfection, l'harmonie, le sens et le but [...]»; à partir du moment où disparaissent «toutes les causes formelles et finales» pour être remplacées par «des causes efficientes et même matérielles». ${ }^{8}$ La persistance tardive, dans la pensée biologique, de schèmes d'explication téléologiques, la difficulté à «mathématiser» l'anatomie et la physiologie (en dépit des efforts de Borelli en particulier), l'orientation de la médecine vers l'efficacité thérapeutique bien plus que vers l'effort spéculatif pour connaître les corps, le recours croissant à l'histoire naturelle présentée comme une approche descriptive et anecdotique de la nature, la permanence de nombreuses références à la philosophie aristotélicienne chez des médecins aussi importants que William Harvey, constitueraient autant d'explications du fait qu'il ait fallu attendre le XIX ${ }^{\mathrm{e}}$ siècle pour pouvoir enfin parler d'une «révolution»en médecine. ${ }^{9}$

\section{Les signes d'un changement}

Depuis le constat opéré en 1990 par Harold J. Cook, la situation a cependant changé. Premièrement, l'idée même de «révolution scientifique» a fait l'objet de réinterprétations et de relectures importantes. La question d'une «essence» de la révolution scientifique, c'est-à-dire d'un moment bien identifié temporellement, permettant selon des critères établis de départager des domaines de scientificité et de rationalité et d'en exclure d'autres, a été largement battue en brèche. L'image d'une «révolution scientifique» contemporaine des découvertes réalisées dans le domaine des sciences physiques et mathématiques dans la première moitié du XVII ${ }^{\mathrm{e}}$ siècle, s'est largement «décentrée», pour faire place à une conception plus ouverte et pluraliste du progrès de la connaissance, dans laquelle les différentes sciences ne progressent pas nécessairement au même rythme, mais communiquent les unes avec

8 Koyré 1968 (1991), 30.

9 Gillispie 1960, 57-59. 
les autres. ${ }^{10}$ Le modèle kuhnien ${ }^{11}$ d'une rupture épistémologique, qui aurait permis à de nouveaux paradigmes de s'imposer en médecine contre les modèles de pensée anciens (la médecine galénique, la médecine chimique d'inspiration paracelsienne), a lui aussi montré ses limites, grâce aux travaux de W. Pagel, C. Webster et A. Debus, qui ont exhibé le rôle prépondérant de la magie, de l'alchimie et de la chimie dans le renouveau et les transformations de la médecine entre la fin de la Renaissance et la période moderne. ${ }^{12}$ Les travaux de Lester King ont montré, dès les années 70, qu'il n'était pas possible de parler d'un abandon de la doctrine des formes et des qualités, de la téléologie et du dynamisme, pour décrire le chemin vers les «lumières médicales» qui s'accomplit entre le XVII ${ }^{\mathrm{e}}$ et le XVIII ${ }^{\mathrm{e}}$ siècles. ${ }^{13}$

Deuxièmement, il existe désormais des ouvrages consacrés à évaluer la nature et le rôle spécifiques de la révolution biologique ou médicale. ${ }^{14}$ Les historiens des sciences et de la philosophie se sont en outre attachés à mener une investigation plus systématique du renouveau de l'anatomie, de la physiologie et de la médecine et de leur réception philosophique, que ce soit à partir des œuvres des anatomistes de la fin de la Renaissance (Vésale, Paré, Du Laurens) ou des médecins de la période moderne (Harvey, Riolan, Glisson, Descartes, Regius, Sydenham, Locke pour ne prendre que quelques exemples).$^{15}$ La réception philosophique de la découverte de la circulation sanguine et les relectures mécanistes auxquelles elle a donné lieu chez Descartes, ont fait l'objet d'études importantes (French 1994) et ont entraîné des relectures du corpus cartésien lui-même (à partir des enjeux des discussions avec Plempius et Froidmont sur le battement du cœur et de leurs résurgences dans la Philosophia naturalis de Regius, par exemple); les implications sociales, politiques et religieuses des travaux des médecins ont aussi été mises en évidence dans l'ouvrage édité par A. Wear et R. French (1989). Des coups de sonde précieux ont aussi permis d'évaluer la nature des changements qui s'opèrent en fonction de contextes nationaux spécifiques. ${ }^{16}$ La présence et le rôle des médecins dans les institutions savantes et leur rôle dans la diffusion et la communication des connaissances en Europe au sein de la République des Lettres sont maintenant reconnus (Académie Royale des Sciences en France, Royal Society en Angleterre). ${ }^{17}$

10 Cunningham/Williams 1993; Osler 2000; Lindberg/Westman 1990; Jacob 2010.

11 Kuhn 1962.

12 Debus 1977; Pagel 1967 et 1982; Webster 1975.

13 King 1970.

14 Grmek 1990. Plus récemment on mentionnera Arquiola/Montiel 2012, la traduction en français de l'ouvrage initialement paru en espagnol, centré sur la France et l'Allemagne.

15 Voir en particulier Duchesneau 1973; Anstey 2011; Mandressi 2003.

16 Voir en particulier Cook 1984; Porter/Teich 1992; Wear 2000.

17 Voir Boas Hall 1991; Hunter 1989. 
L'exemple italien, encore peu étudié, est ici particulièrement intéressant, dans la mesure où la médecine y joue le rôle d'un révélateur de la complexité des processus de construction de la rationalité moderne au sein de contextes différents. En apparence, les trois Académies, «l'Accademia del Cimento» à Florence, «l'Accademia dei Lincei» à Rome et «l'Accademia degli Investiganti» à Naples, se réclament toutes du même héritage galiléo-cartésien. Mais en réalité, aussi bien stratégiquement que tactiquement, elles offrent des visages très divers. La particularité géographique de Naples, soumise à toutes sortes de phénomènes naturels uniques (volcanisme, tremblement de terres, bradyséisme,épidémies, etc.), permet par exemple de discerner concrètement les processus de rationalisation. La polémique autour des risques indissociables de la culture du lin, près du lac d'Agnano, pousse les autorités sanitaires de la ville à considérer le rôle des exhalaisons dans les épidémies de fièvre, voire dans la diffusion de la peste. Le parti des novatores se saisit de cette peur superstitieuse et en fait un des combats de la Modernité à Naples. S'ensuivent un ensemble d'expérimentations et de textes polémiques très divers (rapports d'expériences, correspondance, pamphlets, etc.) dans lesquels des médecins comme Tommaso Cornelio, Leonardo di Capua et Lucantonio Porzio, jouent un rôle décisif. ${ }^{18}$

Troisièmement, les oppositions tranchées - qu'elles portent sur des périodes temporelles (anciens/modernes), sur des modèles de connaissance (empirisme/rationalisme), des courants de pensée (aristotélisme et scolastique d'un côté/cartésianisme de l'autre) - et qui ont largement contribué à rendre la révolution scientifique «introuvable $»^{19}$ ont elles aussi été largement remises en cause depuis ces dernières années. ${ }^{20}$ Parmi celles-ci, l'opposition entre, d'un côté, des sciences physiques ayant adopté unilatéralement un modèle d'explication mécaniste de la nature, et, de l'autre côté, des sciences du vivant encore marquées par des schémas de pensée téléologiques et finalistes, se trouve là encore soumise à une sérieuse réévaluation depuis ces dernières décennies.

Le gain théorique, incontestable, d'une telle réévaluation, est la réintégration du mécanisme et de la téléologie eux-mêmes, dans des schémas explicatifs plus complexes, rendant mieux raison des transformations de fond qui affectent la médecine, dans le lien étroit qu'elle entretient avec les autres champs du savoir:

18 Girard, 2011 et 2015.

19 Duris 2010.

20 Sur la remise en question de l'opposition entre rationalisme et empirisme, voir Cook 1984, 63-80. Sur la question de l'empirisme dans la science moderne et en médecine, voir Wolfe/Gal 2010. Sur l'importance de la notion d'historia pour comprendre le sens de l'empirisme, voir Pomata/Siraisi 2005 et en particulier l'article de Pomata, 105-146. 
Dans le domaine de la physiologie, une part, l'interprétation téléologique se trouve sans doute «progressivement» réduite en interprétation positive des caractéristiques fonctionnelles des phénomènes qui se présentent à l'analyse. Mais nous ne voudrions pas trop accorder à ce qui n'est peut-être que l'«épiphénomène» épistémologique de transformations plus profondes. ${ }^{21}$

Les travaux de Domenico Bertoloni Meli sur Marcello Malpighi donnent un bon exemple des résultats féconds auxquels une telle révision méthodologique permet d'aboutir, non seulement pour la médecine, mais également pour l'histoire de l'anatomie, pour l'histoire des sciences et pour la philosophie. Sans l'examen du contexte géographique, intellectuel et historique de l'acquisition et de la transmission du savoir anatomique de Malpighi, sur les organes humains, les vers à soie et les plantes, on ne peut comprendre l'interaction qu'elles entretiennent avec les philosophies expérimentales et mécanistes, avec l'histoire naturelle et avec la pratique médicale. ${ }^{22} \mathrm{~A}$ cela, il faut ajouter la nécessaire prise en considération des interactions entre l'élaboration des théories physiologiques et le matériau, utilisé et représenté (sur les planches anatomiques, notamment) dans la pratique médicale. ${ }^{23}$

\section{Quels «modèles» pour penser le vivant?}

Comme la chimie et les sciences du vivant, la médecine appartient aux sciences non mathématiques qui s'intègrent difficilement dans les grands panoramas de la révolution scientifique dessinés par Burtt, Dijksterhuis, Koyré, Maier, Butterfield ou Kuhn, qui mettent l'accent sur la mécanisation et la mathématisation de la nature. L'importation de concepts et d'opérations mathématiques, qui a permis à la physique de progresser, ne va pas de soi dans le domaine médical, et certains médecins contestent l'idée selon laquelle la mathématisation et la mécanisation constitueraient la seule manière de faire de la médecine une science ou de définir une véritable méthode en médecine. Mathématiques et mécanisme constituent-ils véritablement les seuls modèles possibles pour penser un progrès dans la connaissance et la guérison du corps?

Dès la seconde partie du XX $\mathrm{X}^{\mathrm{e}}$ siècle, l'œuvre de George Canguilhem a permis d'engager une réflexion sur l'usage que les médecins eux-mêmes faisaient des «modèles» du corps. En ce qui concerne le mécanisme en particulier, Canguilhem a mis en évidence l'existence d'un usage analogique

21 Duchesneau 2012, 19.

22 Bertoloni Meli 2011.

23 Bertoloni Meli 2006. 
et heuristique du «modèle mécaniste» en biologie: «The Cartesian animalmachine remained a manifesto, a philosophical war-machine, so to speak; it did not constitute the programme, scheme, or plan of construction of any particular description of function or structure. ${ }^{24}$ Comparer une partie de l'organisme ou l'organisme à une machine, ce n'est pas nécessairement vouloir le réduire à une machine. En ce sens, et comme l'a montré R. Andrault au sujet de Sténon et de son Discours sur l'anatomie du cerveau (1664), ${ }^{25}$ il faut envisager avec prudence les déclarations des anatomistes qui semblent revendiquer leur filiation avec Descartes en comparant le corps ou une de ses parties (le cerveau par exemple) à une machine.

Ensuite, et depuis les travaux de F. Duchesneau, il n'est plus possible de faire comme si le «mécanisme» correspondait au seul modèle possible d'explication du vivant. Il n'y a pas un modèle du corps ou du vivant aux $\mathrm{XVII}^{\mathrm{e}}$ et $\mathrm{XVIII}^{\mathrm{e}}$ siècles, mais plusieurs, comme en témoignent les notions d'intégration corporelle chez Spinoza, de «principe hylarchique» ou de «nature plastique» chez More $^{26}$ et Cudworth - qui discutent tous deux les limites du mécanisme cartésien - ou bien encore d'«animisme» chez Perrault et Stahl. ${ }^{27}$

Ces différents modèles trouvent leurs répercussions en philosophie, laquelle influe en retour sur les sciences du vivant. En suivant en partie un modèle inspiré de Malpighi, la théorie leibnizienne de la substance conditionne ainsi la détermination du concept du vivant comme «machine de la nature», et la science dynamique participe de l'élaboration du nouveau concept d'«organisme». La science leibnizienne du vivant s'élabore ainsi dans un dialogue constant avec les figures majeures de la médecine de son temps (Hartsoeker, Stahl, Hoffmann, Bernouilli, Michelotti et d'autres encore). Et elle est ensuite intégrée dans les théories sur la génération développées par Conti, Vallisneri puis Bourguet. ${ }^{28}$ En retour, on peut prendre parfaitement au sérieux l'affirmation leibnizienne selon laquelle le monde doit être compris dans des termes fondamentalement biologiques, la biologie venant alors apporter des réponses aux questions métaphysiques les plus épineuses. ${ }^{29}$

Du côté de ceux qui les discutent et les utilisent, ces modèles sont plutôt présentés comme des «opinions» (Willis 1659) ou des «hypothèses» (Locke

24 Canguilhem 1961, 510.

25 Andrault 2009, 55-63.

26 Smith 2009, 307-332.

27 Duchesneau 1998.

28 Duchesneau 2010.

29 Smith 2011. 
1689), ${ }^{30}$ dont il convient de peser attentivement et prudemment les avantages ou les insuffisances, et qu'il est possible, aussi, selon certains philosophes naturels, de combiner pour mieux appréhender la complexité du corps vivant et des phénomènes naturels. Le médecin naturaliste Walter Charleton par exemple, longtemps présenté dans la littérature critique comme figure exemplaire de la conversion de la médecine chimique helmontienne à l'iatromécanisme, ${ }^{31}$ revendique en réalité une méthode éclectique, combinant différentes approches du corps humain. ${ }^{32}$ Les médecins chimistes, présentés comme les rivaux et les adversaires des partisans du mécanisme en médecine (les iatromécanistes) ne sont pas les derniers à avoir recours au modèle de l'horloge pour progresser dans l'explicitation des fonctions vitales et la compréhension du corps humain, en atteste le cas du médecin chimiste George Castle dans son ouvrage The Chemical Galenist (1667). ${ }^{33}$ L'hypothèse corpusculaire doit, selon Boyle, être envisagée de manière différente en médecine et n'invalide pas nécessairement le recours aux remèdes spécifiques des galénistes, dès lors que l'on admet qu'il n'est pas possible d'accéder à une connaissance parfaite des causes des maladies ni de rendre compte de tous les effets observés empiriquement dans les corps (Boyle 1685). Comme le souligne Locke dans son compte-rendu The Reconcileableness of Specifick Medicines to the Corpuscular Philosophy, Boyle attire notre attention sur «une chose» qui est «de la dernière conséquence dans la Médecine et dans la Physique»: à savoir, «qu'il ne faut pas regarder le corps d'un homme qui est en vie, comme une simple statue composée d'un amas de chair, de sang, d'os, de graisse, de nerfs, de veines, d'artères etc.». ${ }^{34}$

Enfin, on a insisté, depuis les années 2000, sur la manière dont la physiologie et la médecine permettent d'interroger les limites d'un modèle mécaniste d'explication des phénomènes naturels, dès lors que ce dernier entend rendre compte de l'ensemble de ces phénomènes et intégralement à partir de la seule considération des figures, et du mouvement des particules de matière. Stephen Gaukroger ${ }^{35}$ a pointé de manière particulièrement éclairante cette difficulté au sujet de Descartes tout d'abord, et de ses efforts pour rendre compte de ce qu'il nomme les «goal-directed processes», qu'il s'agisse du développement du fœtus chez l'homme ou d'une forme de perception cognitive chez les animaux - un des motifs qui conduit d'ailleurs un méde-

30 Anstey 2011.

31 Brown 1981.

32 Booth 2005.

33 Cet ouvrage est évoqué par Debus 2001,141.

34 Locke dans Le Clerc 1687.

35 Gaukroger 2000, 383-400. 
cin et philosophe naturel comme Thomas Willis à discuter Descartes et l'approche mécaniste du vivant dans son De Anima Brutorum (London, 1672). Certes la philosophie naturelle mécaniste s'est fixé comme principal objectif au XVII ${ }^{e}$ siècle, l'élimination de la téléologie, mais il est loin d'être sûr qu'elle y soit parvenue. Il apparaît en outre nécessaire de tenir compte et d'étudier les controverses et disputes, à l'issue desquelles une hypothèse semble s'imposer comme la plus probable. Les transcriptions et le renouveau des recherches autour des manuscrits médicaux de Locke ont en particulier permis, ces dernières années, ${ }^{36}$ de montrer que le primat accordé à l'hypothèse corpusculaire dans l'Essay résultait d'un examen attentif des différentes hypothèses présentes dans le champ de la philosophie naturelle de la seconde partie du XVII ${ }^{\mathrm{e}}$ siècle, qu'il s'agisse de convoquer les ferments des chimistes ou de se pencher sur les principes de la médecine galénique. L'étude clinique et la description de l'opération du premier comte de Shaftesbury, retranscrites par Anstey et Principe, ne nous offrent pas seulement un document historique unique sur la naissance de la méthode clinique à l'époque moderne. Elles permettent en outre de comprendre comment l'observation des vers parasites chez les hommes et les animaux (cf. les recherches du botaniste John Ray) a pu conduire Locke à considérer dans sa correspondance en 1694, à considérer que le débat sur la génération spontanée ne devait pas être considéré comme totalement infondé. ${ }^{37}$

La nature des liens entre mécanisme et téléologie est donc plus complexe que celle qui a été véhiculée par une certaine lecture de Descartes et du cartésianisme. Tout d'abord, les travaux menés ces dernières décennies autour de la philosophie naturelle de William Harvey ont permis de prendre la mesure de l'effort accompli par Descartes pour intégrer dans son approche mécaniste de la nature un phénomène vital qui pourtant résiste à ce type d'explication. ${ }^{38}$ L'explication harveyenne du mouvement du sang fait appel à une force de contraction du cœur (le mécanisme appliqué à la médecine implique au contraire de nier tout pouvoir d'attraction entre les parties d'un organe), elle reconnaît elle-même sa dette à l'égard d'Aristote et fait intervenir l'âme pour rendre compte du sang comme principe de vie. Pourtant, c'est souvent à partir de sa présentation par Descartes dans le Discours de la méthode que le public lettré et savant du XVII ${ }^{e}$ siècle a pris connaissance de la découverte de Harvey. Cela peut sans doute expliquer pourquoi les ouvrages consacrés à Harvey ont souvent présenté sa philosophie comme mécaniste, bien que

36 Walsmey 1998; Anstey/Principe 2011.

37 Locke à Sloane, 14 septembre 1794; Beer 1976-1989, 5, 128.

38 French 1994 et 2003, 182-184. 
cette approche ait été contestée dès les travaux de W.Pagel ${ }^{39}$ et de R.French $^{40}$, de R. Ariew ${ }^{41}$ ou de T. Verbeek ${ }^{42}$. Ou que certains historiens des sciences présentent Descartes comme celui qui aurait débarrassé l'explication de la circulation du sang des éléments «vitalistes» et «occultes» qui subsistaient encore chez Harvey. ${ }^{43}$ A l'inverse, le renouveau des lectures de Harvey a reposé, chez Descartes même, la question de l'éventuelle persistance de schémas explicatifs aristotéliciens au sein du mécanisme. Plusieurs attitudes sont alors possibles et diversement fécondes. On peut tout d'abord réévaluer historiquement l'importance de l'aristotélisme au moment même où le traité de L'Homme et la Description du corps humain semblent réécrire le De Anima, comme Le Monde réécrit la Physique. ${ }^{44}$ On peut ensuite chercher, dans les œuvres des médecins ayant combattu aux côtés de Descartes contre les formes substantielles, des arguments mécanistes montrant que justement, l'explication cartésienne du mouvement du cœur ne réintroduit pas ces dernières. Au fur et à mesure des réécritures des passages concernant ces questions dans le livre IV de sa Philosophia naturalis, Regius particularise ainsi les critères mécanistes (l'agitation violente et en tous sens des esprits animaux) pour endiguer toute tentation de recourir à un principe d'une nature autre que matérielle pour rendre raison de ce mouvement. Mais par là même, il montre que Descartes ne l'avait pas fait, ou pas suffisamment, au regard de ses adversaires scolastiques, comme du point de vue de ses partisans mécanistes. Quel que soit le parti adopté, la rectification de l'interprétation des schèmes explicatifs valables pour un philosophe ou un médecin rejaillit donc à la fois sur l'œuvre propre de ce philosophe ou de ce médecin, et sur celles dont il s'inspire et dont son interprétation a pu, parfois (c'est le cas de Descartes pour Harvey) supplanter la lecture de l'œuvre originelle, dans l'histoire de sa réception. Les histoires de la philosophie et de la médecine sont ainsi, partiellement, des histoires à la fois construites et emboîtées les unes dans les autres.

Il est ainsi possible de conclure en revenant au plus célèbre des historiens de la philosophie française au XIX ${ }^{\mathrm{e}}$ siècle: Victor Cousin. Dans son Histoire générale de la philosophie depuis les temps les plus anciens jusqu'au $X I X^{e}$ siècle, ${ }^{45}$ le fondateur de l'école spiritualiste éclectique entend répondre

39 Pagel 1967 et 1976.

40 French 1994.

41 Ariew 1999.

42 Verbeek 1992.

43 Voir par exemple Westfall 1971, 93.

44 Des Chene 1996.

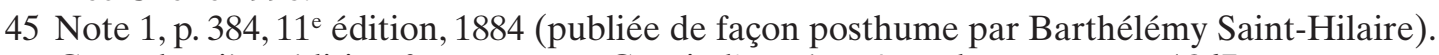
Cette dernière édition fut revue par Cousin l'année même de sa mort, en 1867. 
aux accusations leibniziennes qui autorisent, via le bannissement radical des causes finales, un rapprochement dangereux entre Descartes et Spinoza. Pour cela, Cousin préserve la spécificité du projet cartésien en physique, tel qu'il s'explicite dans l'article 28 de la première partie des Principes de la philosophie, mais pour mieux montrer que Descartes ne fut pas insensible à la nécessité de reconnaître une forme de finalité traduisant, à même les choses vivantes, l'exercice de la sagesse divine. Alors que tout le projet cartésien vise à «décrire» le corps humain, Cousin puise ainsi ses arguments, non dans le lexique de Descartes, mais dans celui que Clerselier ajouta au texte de L'Homme pour lui fournir une table des matières, dans l'édition posthume de 1664: le vocabulaire de l'«usage». Le subterfuge consiste ainsi à intercaler entre Leibniz et Descartes un nouveau prisme implicite: celui de l'éditeur zélé du philosophe du cogito, soucieux de répondre aux attaques matérialistes dont ce dernier faisait l'objet, mises à l'index à l'appui, au début des années 1660, afin d'ériger la lecture de Clerselier en seule interprétation valable du projet de Descartes en physiologie.

On le voit: non seulement les mutations de la médecine ne sont pas indépendantes de celles qui affectent les autres champs du savoir, à commencer par la philosophie. Mais, plus encore, on peut difficilement comprendre les enjeux des principales hypothèses discutées dans le domaine de la philosophie naturelle, et leurs répercussions sur un plan métaphysique (la question de la nature du corps, de l'âme, de leur rapport, la possibilité d'une connaissance de la nature et des substances, les notions de mécanisme et de force, etc.) sans repartir des discussions et des débats entre médecins et philosophes. Ces débats sont divers et féconds: ils portent aussi bien sur la méthode (methodus medendi), que sur la manière de penser le rapport entre expérience et raison, sur les hypothèses ou les opinions en présence pour tenter de progresser dans l'investigation de la nature des corps, et plus particulièrement du corps vivant. L'étude de cette «tradition médico-philosophique» ${ }^{46}$ moderne apparaît donc nécessaire dès lors que l'on se donne comme objectif non pas de reconstituer a posteriori une histoire mythique qui consacrerait le triomphe d'un modèle de rationalité contre un autre, mais plutôt de tenter de retracer la genèse, mouvementée et conflictuelle, de l'apparition d'une diversité de schèmes explicatifs qui ne s'excluent pas les uns les autres, mais tentent de se combiner pour rendre compte d'un objet complexe: le corps vivant.

46 Selon la définition donnée par Pigeaud 1981. 


\section{Bibliographie}

Textes cités

Andrault, Raphaële, Nicolas Sténon, Discours sur l'anatomie du cerveau (Paris 2009) Anstey, Peter, John Locke and Natural Philosophy (Oxford 2011)

Anstey, Peter/Lawrence Principe, "John Locke and the Case of Anthony Ashley Cooper", Early Science and Medicine 16 (2011) 379-503

Ariew, Roger, Descartes and the Last Scholastics (Ithaca/London 1999).

Arquiola, Elvire/Luis Montiel, La médecine en révolution. Sciences et philosophie de la nature au tournant du XVIII e et du XIX $X^{e}$ siècles (Paris 2012)

Beer, E.S. de, The correpondence of John Locke, 8 vols. (Oxford 1976-1989)

Bertoloni Meli, Domenico, Thinking with Objects. The transformation of Mechanics in the Seventeenth century (Baltimore 2006)

Bertoloni Meli, Domenico, Mechanism, Experiment, Disease. Marcello Malpighi and Seventeenth-Century Anatomy (Baltimore 2011)

Boas Hall, Mary, Promoting Experimental Learning, Experiment and the Royal Society 1660-1727 (Cambridge 1991)

Booth, Emily, "A Subtle and Mysterious Machine”. The Medical World of Walter Charleton (1619-1707) (Dordrecht 2005)

Brown, Theodore, The Mechanical Philosophy and the "Animal Oeconomy" (New York 1981)

Butterfied, Herbert, The Origins of Modern Science, 1300-1800 (London 1965)

Canguilhem, Georges, "The Role of Analogies and Models in Biological Discovery", in: Alistair C. Crombie (éd.), Scientific Change. Historical Studies in the intellectual, social and technical conditions for scientific discovery and technical invention from antiquity to the present. Symposium on the History of Science (London 1961) 507-541

Cook, Harold J., "Physick and Natural History in Seventeenth Century England", in: Peter Barker/Roger Ariew, Physick and Natural History in Seventeenth Century England, Revolution and Continuity. Essays in the History and Philosophy of Early Modern Science (Washington 1984) 63-80

Cook, Harold J., "The new philosophy and medicine in seventeenth-century England", in: David C. Lindberg/Robert S. Westman (éds), Reappraisals of the Scientific Revolution (Cambridge 1990) 397-436

Cousin, Victor, Histoire générale de la philosophie depuis les temps les plus reculés jusqu'au XIX $X^{e}$ siècle (Paris 1884)

Cunningham, Andrew/Perry Williams, "Decentring the Big Picture: the Origins of Modern Science and the Modern Origins of Science", British Journal of the History of Science 26 (1993) 407-432

Debus, Allen G., The chemical philosophy: Paracelsian science and medicine in the sixteenth and seventeenth centuries (New York 1977)

Debus, Allen G., Chemistry and Medical Debate van Helmont to Boerhaave (New York 2001)

Des Chene, Dennis, Physiologia. Natural philosophy in late Aristotelian and Cartesian thought (Ithaca/London 1996)

Dijksterhuis, Eduard Jan, The Mechanization of the World Picture: Pythagoras to Newton (orig. néerlandais Amsterdam 1950, première éd. anglaise New York 1961, rééd. anglaise New York 1986) 
Duchesneau, François, L'empirisme de Locke (The Haye 1973)

Duchesneau, François, Les modèles du vivant de Descartes à Leibniz (Paris, 1998)

Duchesneau, François, Leibniz, le vivant et l'organisme (Paris, 2010)

Duchesneau, François, La physiologie des Lumières. Empirisme, modèles et théories (Paris 2012; première éd. Den Haag/London/Boston 1982)

Duris, Pascal, «L'introuvable révolution scientifique. Francesco Redi et la génération spontanée», Annals of Science 67,4 (2010) 431-455

French, Roger, William Harvey's natural philosophy (Cambridge 1994)

French, Roger, Medicine Before Science: the rational and learned doctor from the middle ages (Cambridge 2003)

French, Roger/Andrew Wear (éds), The Medical Revolution of the $17^{\text {th }}$ century (Cambridge 1989)

Gaukroger, Stephen, "The resources of a mechanist physiology and the problem of goal-directed processes”, in: Stephen Gaukroger/John Schuster/John Sutton (éds), Descartes' Natural Philosophy (London/New York 2000) 383-400

Gillispie, Charles C., The Edge of Objectivity: an Essay in the History of scientific ideas (Princeton 1960) 58-73

Grmek, Mirko D., La première révolution biologique: réflexions sur la physiologie et la médecine du XVII ${ }^{\mathrm{e}}$ siècle (Paris 1990)

Grmek, Mirko D., «Le concept de maladie», in: Histoire de la pensée médicale en Occident (Paris, 1997) 157-176

Hunter, Michael, Establishing the new science, the experience of the early Royal Society (Woodbridge 1989)

Jacob, Margaret J. (éd.), The Scientific Revolution. A Brief History with Documents (Boston/New York 2010)

King, Lester S., The Road to Medical Enlightenment, 1650-1695 (New York 1970)

Koyré, Alexandre, Études galiléennes (Paris 1966; rééd. 2001)

Koyré,Alexandre, «Sens et portée de la synthèse newtonienne», in: Alexandre Koyré, Études newtoniennes (Paris 1968; rééd. 1991) 27-49

Kuhn, Thomas, The Structure of Scientific Revolution (Chicago 1962)

Lindberg, David C./Robert S. Westman (éds), Reappraisals of the Scientific Revolution (Cambridge 1990; version digitale 2004)

Locke, John, De Specificorum Remediorum cum corpusculari Philosophia Concordia, cui accessit Dissertation de varia simplicium Medicamentorum utilitate, usuque, in: Jean Le Clerc, Bibliothèque Universelle et Historique de l'année 1686 (Amsterdam 1687) 263-280

Locke, John, Essay Concerning Human Understanding, ed. Nidditch (Oxford 1975)

Mandressi, Raphael, Le regard de l'anatomiste. Dissections et invention du corps en Occident (Paris 2003)

Osler, Margaret J. (éd.), Rethinking the Scientific Revolution (Cambridge 2000)

Pagel, Walter, William Harvey's Biological Ideas (Basel/New York 1967)

Pagel, Walter, New Lights on Harvey (Basel/New York 1976)

Pagel, Walter, Joan Baptista von Helmont, reformer of science and medicine (Cambridge 1982)

Pigeaud, Jackie, La maladie de l'âme: étude sur la relation de l'âme et du corps dans la tradition médico-philosophique antique (Paris 1981)

Pomata, Gianna, "Praxis historialis: The Uses of Historia in Early Modern Medicine", 
in: Gianna Pomata/Nancy G. Siraisi (éds.), Historia. Empiricism and Erudition in Early Modern Europe (Cambridge 2005) 105-46

Porter, Roy/Mikulas Teich (éds.), Scientific Revolution in National Context (New York 1992).

Rothschuh, Karl Eduard, Geschichte der Physiologie (Berlin 1953)

Smith, Justin E.H., "Descartes and Henry More on Living Bodies", in: Vlad Alexandrescu (éd.), Branching off. The Early Moderns in Quest for the Unity of Knowledge (Bucharest 2009) 307-332

Smith, Justin E.H., Divine Machines. Leibniz and the Sciences of Life (Princeton 2011)

Temkin, Owsei, Galenism: rise and decline of a medical philosophy (Ithaca (NY) 1973)

Verbeek, Theo, Descartes and the Dutch. Early Reactions to Cartesian Philosophy 1637-1650 (Carbondale/Edwardsville 1992)

Walsmey, Jonathan Craig, John Locke's Natural Philosophy (1632-1671) (PhD Diss., King's College London 1998, reformaté 2008)

Wear, Andrew, Knowledge and Practice in English Medicine, 1550-1680 (Cambridge 2000)

Webster, Charles, The Great Instauration, Science, Medicin and Reform, 1626-1660 (Duckworth 1975)

Westfall, Richard S., "Biology and the Mechanical Philosophy", in: Richard S. Westfall, The Construction of Modern Science: Mechanisms and Mechanics (Cambridge 1971; réimp. 1977) 82-104

Wolfe, Charles/Ofer Gal (éds), The Body as Object and Instrument of Knowledge. Embodied Empiricism in Early Modern Science (Dordrecht/Heidelberg/London/ New York 2010)

Bibliographie complémentaire

Ariew, Roger/Marjorie Grene (éds), Descartes and his Contemporaries. Meditations, Objections, and Replies (Chicago/London 1995)

Berthier, Auguste-Georges, «Le mécanisme cartésien et la physiologie au XVII ${ }^{\mathrm{e}}$ siècle», Isis 2,1 (Jun. 1914) 37-89; 3,1 (Jan. 1920) 21-58

Bitbol-Hespériès, Annie, Le principe de vie chez Descartes (Paris 1990)

Bloch, Olivier-René (éd.), Images au XIX $X^{e}$ siècle du matérialisme du XVIII ${ }^{e}$ siècle (Paris 1979)

Borghero, Carlo, Les Cartésiens face à Newton. Philosophie, science et religion dans la première moitié du XVIII siècle (Turnhout 2011)

Bos, Erik-Jan (éd.), The Correspondence between Descartes and Henricus Regius (Utrecht 2002)

Breck, Allen D./Wolfgang Yourgrau (éds), Biology, History and Natural History (New York 1972)

Calan, Ronan de, Généalogie de la sensation. Physique, physiologie et psychologie en Europe, de Fernel à Locke (1550-1675) (Paris 2012)

Clarke, Desmond, Descartes' Philosophy of Science (Pennsylvania 1982)

Cohen, Floris H., The Scientific Revolution. A Historiographical Inquiry (Chicago 1994)

Cook, H.J., "Physick and Natural History in Seventeenth-Century England", in: Peter Barker/Roger Ariew (éds), Revolution and Continuity: Essays in the History and Philosophy of Early Modern Science (Washington 1991) 63-80 
Crignon, Claire, «La découverte de la circulation sanguine: révolution ou refonte?», Gesnerus, Swiss Journal of the History of Medicine and Sciences 68,1 (2011) 5-25

Daled, Pierre F., Le matérialisme occulté ou la genèse du sensualisme. Écrire l'histoire de la philosophie en France (Paris 2006)

Des Chene, Dennis, Life's Form. Late Aristotelian Conceptions of the Soul (Ithaca/ London 2000)

Des Chene, Dennis, Spirits and Clocks. Machine and Organism in Descartes (Ithaca/ London 2001)

Dibon, Paul, Regards sur la Hollande du Siècle d'Or (Naples 1990)

Duchesneau, François, «Leibniz et Stahl: divergences sur le concept d'organisme», Studia Leibnitiana 27 (1995) 185-212

Fisher, Saul, Pierre Gassendi's philosophy and science; atomism for empiricists (Leiden 2005)

Frank, Robert J., The Oxford Physiologists: Scientific Ideas and Social Interaction (Berkeley/Los Angeles/London 1980)

Gabbey, Alan, "Henry More and the Limits of Mechanism”, in: Sarah Hutton (éd.), Henry More (1614-1687). Tercentenary Studies (Dordrecht 1990) 19-35

Gaukroger, Stephen, The emergence of a scientific culture: science and the shaping of modernity, 1210-1685 (Oxford 2006)

Girard, Pierre, «Matérialisme et politique: les enjeux de la réception du cartésianisme à Naples à l'Âge classique» Corpus 61 (2011) 113-132

Girard, Pierre, "Comme des Lumières jamais vues». Matérialisme et radicalisme politique dans les premières Lumières à Naples (1647-1744) (Paris, 2015)

Henry, John, "Occult Qualities and the Experimental Philosophy. Active Principles in Pre-Newtonian Matter Theory", History of Science 24 (1986) 335-381

Hoffmann, Paul, «Modèle mécaniste et modèle animiste. De quelques aspects de la représentation du vivant chez Descartes, Borelli et Stahl», Revue des Sciences Humaines 186-187 (1982-1983) 199-211

Kolesnik-Antoine (Antoine-Mahut), Delphine, L'homme cartesien. La «force qu'a l'âme de mouvoir le corps»: Descartes, Malebranche (Rennes 2009)

Kolesnik-Antoine (Antoine-Mahut), Delphine, «Les voies du corps. Schuyl, Clerselier et La Forge lecteurs de L'Homme de Descartes», in: Francesco Toto/Roberto Finelli (eds): Per un nuovo Materialismo. Consecutio temporum. Rivista critica delle postmodernitá 2 (2012) 118-128

Lüthy, Christoph/John E. Murdoch/William R. Newman (éds), Late Medieval and Early Modern Corpuscular Matter Theories (Leiden/Boston/Köln 2001)

Osler, Margaret J., "How mechanical was the mechanical philosophy", in: Christoph Lüthy/John E. Murdoch/William R. Newmann (éds), Late Medieval and Early Modern Corpuscular Matter Theories, (Leiden/Boston/Köln 2001) 423-439

Shackelford, Jole, "Seeds with a Mechanical Purpose: Severinus's Semina and Seventeenth Century Matter Theory", in: Allen G. Debus/Michael T. Walton (éds), Reading the Book of Nature, The Other side of the Scientific Revolution (Kirksville 1998) 15-44

Siegel, Rudolph Edward, Galen's System of Physiology and Medicine. An Analysis of his Doctrines and Observations on Bloodflow, Respiration, Humors and Internal Diseases (Basel/New York 1968) 
Smith, Justin E.H. (éd.), The Problem of Animal Generation in early Modern Philosophy from Descartes to Kant (Cambridge 2006)

Westfall, Richard S., The Revolution in Science, 1500-1750 (London 1983)

Westfall, Richard S., "The Scientific Revolution Reasserted", in: Margaret J. Osler (éd.), Rethinking the Scientific Revolution (Cambridge 2000) 41-55

Williams, Elizabeth A., The physical and the moral. Anthropology, physiology, and philosophical medicine in France 1750-1850 (Cambridge 1994) 